\title{
Schwinger Particle-Production Mechanism for a Finite-Length Flux Tube with Transverse Confinement
}

\author{
Cheuk-Yin Wong \\ Oak Ridge National Laboratory, Oak Ridge, TN 37831 \\ Ren-Chuan Wang \\ University of Science and Technology of China, Hefei, China \\ Jian-Shi Wu \\ Fayetteville State University, Fayetteville, NC 28301
}

(June 28, 2021)

\begin{abstract}
Previous results for the pair production probability in a strong electric field with a finite longitudinal separation are generalized to the case of a finitelength flux tube with transverse confinement. The threshold length of the flux tube, below which pair production cannot occur, increases as a result of transverse confinement.
\end{abstract}

Typeset using REVTEX 


\section{INTRODUCTION}

The Schwinger particle production mechanism has found applications in many branches of physics [1]- [7]. It was originally derived [1] using the Green's function and gauge invariance for the case where the strength of the Abelian electric field is a constant in the whole configuration space. For many physics applications, the field strength exists only in a limited region of space. For example, in quantum chromodynamics (QCD), the color flux tube is a useful concept, as it explains many qualitative features of nonperturbative phenomena such as confinement, Reggeon trajectories, and particle production [2]- [7]. The potential energy of a flux tube is linearly proportional to their separation. Hence, we can approximate the field between the particle-antiparticle pair by a constant Abelian electric field, as in the case studied by Schwinger. The electric field strength is non-zero only in a finite spatial region. It is of interest to consider the case of the Schwinger production mechanism for which the electric field is restricted to a finite longitudinal and transverse region.

Previously, Wang and Wong ( [3], which we shall call I) studied the Schwinger particle production mechanism for the case where an electric field exists only between two parallel condenser plates at a finite longitudinal separation. The transverse dimension is taken to be infinite. The Klein-Gordon and the Dirac equations are solved in this field. The transmission amplitude, which is related to the probability for pair production, was worked out and written explicitly in terms of parabolic cylinder functions for bosons and confluent hypergeometric functions for fermions [3]. It is found that pair-production occurs only when the longitudinal separation is greater than $m_{0} / \sigma$ where $m_{0}$ is the rest mass of the produced particle and $\sigma$ is the string tension. The pair-production probability is much suppressed when the separation just exceeds the threshold value. The probability also shows oscillations due to the finite separation. The same problem was examined by Martin and Vautherin using Schwinger's Green's function formalism and Balian and Bloch's multiple reflection expansion method [8]. Martin and Vautherin also studied the effect for the case with a transverse boundary. 
Subsequently, Pavel and Brink [9] introduced the scalar potential $m(r)$ in the transverse direction to study a flux tube of finite transverse radius. They examined a flux tube with a sharp transverse boundary and infinite longitudinal length, which was also studied by Sailer et al. [10] and Schöfeld et al. [11]. They obtained pair-production probability which was found to be suppressed due to the finite radius of the tube. Sailer et al. [10] and Schöfeld et al. [11] also calculated the transverse momentum distribution for a flux tube with a sharp boundary. In a recent work, Gatoff and Wong [12] studied the low $p_{T}$ spectra in terms of a smooth-varying transverse potential. The Dirac equation was solved by using the method of separation of variables of Pavel and Brink [9]. The effect of including an additional scalar potential rising linearly with the absolute magnitude of the longitudinal coordinate was investigated by Warke and Bhalerao [13,14]. Pair production in the color dielectric model with a smooth transverse boundary has been investigated by Flintoft and Birse [15.

Previous works of Schwinger mechanism with transverse confinement deals with a color flux tube of infinite longitudinal length. We wish to investigate in this paper the case of the Schwinger particle production mechanism for a finite-length flux tube with transverse confinement for both bosons and fermions. As the pair production probability depends on the transmission amplitude for longitudinal incident waves, it is necessary to introduce the incident, the reflected and the transmitted waves. In Refs. [9] and [12], no explicit prescription was given to separate the longitudinal incident waves and the transmitted waves. In the work of Pavel and Brink [9] for fermions, the longitudinal component of the wave function are different for different spinor components (see Eq. (3.7) and (3.11) of Ref. [9]) and the formulation of the transmission and reflection of incident waves to obtain the pair production probability was not carried out. We wish to report here a different method of separating variables, by following the representation of our earlier work in Ref. [3]. With this representation, the longitudinal wave function can be completely factorized out from the transverse wave function in the spinor form (see Eqs. (3.2) and (3.4) below). Therefore, we can introduce explicitly the longitudinal incident, reflected and transmitted waves. This method provides a formalism for calculating the transmission amplitude for the general case 
of a flux tube of finite longitudinal length $L$ and a smooth varying transverse confining scalar potential. The separation of the incident and transmitted waves turns out to be just a generalization of the previous results in I (Ref. [3]). The results on the transmission amplitude and reflection amplitude from Ref. [3] can be used directly for the case with transverse confinement by replacing the continuous transverse mass $m_{\perp}$ of I with the discrete eigenvalues when there is a transverse confining potential. Thus, in the application of the Schwinger mechanism for particle production, the proper mass involved in the production process is not the rest mass but the transverse mass, including the effect of the transverse zero-point motion.

\section{BOSONS IN A STRONG FIELD}

We consider first a boson of mass $m_{0}$ in a linear vector potential $A=\left(A_{0}(z), 0,0,0\right)$ where $A_{0}$ depends only on $z$ and is of the form

$$
A_{0}(z)=\left\{\begin{array}{lll}
0 & \text { for } z \leq 0 & \text { (region I) } \\
-\kappa z & \text { for } 0 \leq z \leq L & \text { (region II) } \\
-\kappa L & \text { for } L \leq z & \text { (region III) }
\end{array}\right.
$$

Such a linear potentail arises, for example, in an approximate description of pair production in a flux tube of length $L$ in QCD, where the strength parameter $\kappa$ is related to the string tension $\sigma(\kappa=2 \sigma$ in $[7,3,4])$. We study the case where the transverse motion of the boson is restricted and described by a transverse scalar potential $m(r)$ which includes the rest mass $m_{0}$. The equation of motion of the boson is the Klein-Gordon equation:

$$
\left[(p-A)^{2}-m^{2}(r)\right] \phi(r, \varphi, z)=0
$$

The solution of the equation can be written in the form $\phi(r, \varphi, z)=R_{\nu}(r) e^{i \nu \varphi} f(z)$, which allows the equation to be separated into the set of coupled equations

$$
\begin{gathered}
{\left[-\partial_{z}^{2}+m_{\perp}^{2}-\left(p_{0}-A_{0}\right)^{2}-i \partial_{z} A_{0}(z)\right] f(z)=0,} \\
{\left[-\frac{1}{r} \frac{\partial}{\partial r} r \frac{\partial}{\partial r}+\frac{\nu^{2}}{r^{2}}+m^{2}(r)-m_{\perp}^{2}\right] R_{\nu}(r)=0}
\end{gathered}
$$


where $m_{\perp}$ is the constant of separation and plays the role of the transverse mass. For a given transverse quantum number $\nu$, one solves for the transverse wave function $R_{\nu}(r)$ in Eq. (2.4) with the eigenvalue $m_{\perp}^{2}$, subject to the boundary condition that the wave function $R_{\nu}(r) \rightarrow 0$ as $r \rightarrow \infty$. For the case of a sharp boundary with a radius $r_{0}$, the minimum eigenvalue of $m_{\perp}$ has the value $m_{\perp}=\sqrt{m_{0}^{2}+\left(2.404 / r_{0}\right)^{2}}$, which increases as the size of the flux tube $r_{0}$ decreases. In another example, with $m^{2}(r)=m_{0}^{2}+\sigma^{2} r^{2}$, the lowest value of $m_{\perp}$ is $m_{\perp}=\sqrt{m_{0}^{2}+2 \sigma}$, which contains the transverse zero-point energy. In the special case when the boson is a gluon with a zero rest mass, the gluon acquires an effective mass $m_{\perp}$ as a result of transverse confinement. Finally, for two parallel plates for which $r_{0} \rightarrow \infty$, there is no restriction in the transverse motion. The solution of $R(r)$ contains plane waves in the transverse directions and $m_{\perp}^{2}=m_{0}^{2}+p_{T}^{2}$.

After obtaining the eigenvalue $m_{\perp}$ from the transverse motion, the dynamics of the boson for the longitudinal motion is determined by Eq. (2.3). One notes that Eq. (2.3) is the same as the boson case in I by replacing the continuous transverse mass $m_{\perp}$ in I with discrete $m_{\perp}$. Because of this simple relationship, one can carry out the same steps of derivation so that previous results in I for the transmission and the reflection of an incident wave coming from the right in Region I for two parallel condenser plates can be used directly here.

\section{FERMIONS IN A STRONG FIELD}

To examine the production of fermion-antifermion pairs in a flux tube we study the motion of a fermion in the Abelian gauge field $A_{\mu}=\left(A_{0}(z), 0,0,0\right)$, and the transverse confining potential $m(r)$, as introduced in Section II. The Dirac equation for the fermion in cylindrical coordinates $(r, \varphi, z)$ is:

$$
[\gamma \cdot \Pi-m(r)] \psi(r, \varphi, z)=0
$$

where $\gamma \cdot \Pi=\gamma^{\mu} \Pi_{\mu}$ and $\Pi_{\mu}=p_{\mu}-A_{\mu}$. A fermion and an antifermion are spontaneously produced in this field when a fermion in a negative-energy state tunnels from the negative 
energy continuum to the positive energy continuum [3]. Generalizing the results of Ref. [3], we seek a solution of the Dirac equation (3.1) in the form

$$
\psi(r, \varphi, z)=[\gamma \cdot \Pi+m(r)] \phi(r, \varphi, z)
$$

The equation for $\phi$ is

$$
\left\{\left(p_{0}-A_{0}\right)^{2}-\boldsymbol{p}^{2}-m^{2}(r)+i \alpha^{3} \partial_{z} A_{0}(z)+i\left[\left(\gamma^{1} \partial_{1}+\gamma^{2} \partial_{2}\right) m(r)\right]\right\} \phi(r, \varphi, z)=0
$$

We note that $\left[\alpha^{3}, J_{z}\right]=0$, where $J_{z}=-i \partial / \partial \phi+\sigma_{z} / 2$ is the third component of the angular momentum operator. Furthermore, both $J_{z}$ and $\alpha^{3}$ commute with the operator acting on $\phi(r, \phi, z)$ in Eq. (3.3). Therefore, we may choose the $\phi(r, \varphi, z)$ to be factorized as

$$
\phi_{\eta \nu}(r, \varphi, z)=f_{\eta \nu}(z) \rho_{\eta \nu}(r, \varphi)
$$

with $\rho_{\eta \nu}(r, \varphi)$ to be simultaneous eigenfunctions of $\alpha^{3}$ and $J_{z}$. Upon using the representation in Ref. [3], the eigenfunction of $\alpha^{3}$ satisfying $\alpha^{3} \mu_{\lambda}=\eta_{\lambda} \mu_{\lambda}$ are

$$
\mu_{1}=\frac{1}{\sqrt{2}}\left(\begin{array}{l}
1 \\
0 \\
1 \\
0
\end{array}\right), \quad \mu_{2}=\frac{1}{\sqrt{2}}\left(\begin{array}{c}
0 \\
1 \\
0 \\
-1
\end{array}\right), \quad \mu_{3}=\frac{1}{\sqrt{2}}\left(\begin{array}{c}
1 \\
0 \\
-1 \\
0
\end{array}\right), \quad \mu_{4}=\frac{1}{\sqrt{2}}\left(\begin{array}{l}
0 \\
1 \\
0 \\
1
\end{array}\right) \text {, }
$$

with $\eta_{1,2}=+1$ and with $\eta_{3,4}=-1$. Thus, the eigenfunctions of $J_{z}$ satisfying $J_{z} \rho_{\eta \nu}=$ $\left(\nu+\sigma_{z} / 2\right) \rho_{\eta \nu}$ are

$$
\begin{aligned}
& \rho_{\eta \nu}=g_{1 \nu}(r) e^{i \nu \phi} \mu_{1}-g_{2 \nu}(r) e^{i(\nu+1) \phi} \mu_{2} \text { for } \eta=+1, \\
& \rho_{\eta \nu}=g_{1 \nu}(r) e^{i \nu \phi} \mu_{3}+g_{2 \nu}(r) e^{i(\nu+1) \phi} \mu_{4} \quad \text { for } \eta=-1 .
\end{aligned}
$$

The Dirac equation can be easily separated into the set of equations

$$
\left[-\partial_{z}^{2}+m_{\perp}^{2}-\left(p_{0}-A_{0}\right)^{2}+\eta i \partial_{z} A_{0}(z)\right] f_{\eta \nu}(z)=0
$$




$$
\begin{gathered}
{\left[\boldsymbol{p}_{\perp}^{2}(\nu)+m^{2}(r)-m_{\perp}^{2}\right] g_{1}(r)=i \frac{\partial m(r)}{\partial r} g_{2}(r),} \\
{\left[\boldsymbol{p}_{\perp}^{2}(\nu+1)+m^{2}(r)-m_{\perp}^{2}\right] g_{2}(r)=-i \frac{\partial m(r)}{\partial r} g_{1}(r),}
\end{gathered}
$$

where

$$
\boldsymbol{p}_{\perp}^{2}(\nu)=-\frac{1}{r} \frac{\partial}{\partial r}\left(r \frac{\partial}{\partial r}\right)+\frac{\nu^{2}}{r^{2}}
$$

When $m(r)=$ constant, the two functions are decoupled.

Note that in the transverse direction the positive and negative energy states are degenerate for $\rho_{\eta \nu}(r, \varphi)$, and $\left[\eta m_{\perp} \beta-\boldsymbol{\gamma}_{\perp} \cdot \boldsymbol{p}_{\perp}+m(r)\right] \rho_{\eta \nu}(r, \varphi)$ would automatically produce two different solutions with $\eta= \pm 1$ for the transverse Dirac equation, because of the sign difference in the $m_{\perp}$ term. The advantage of separating the variables in the form of Eqs. (3.2) and (3.4) is that as $f_{\eta \nu}$ is factorized out, the separation of the corresponding components of the wave function in terms of the transmission and reflection amplitudes can be easily carried out. To determine these amplitudes, we match the wave function $f_{\eta \nu}(z)$ and $f_{\eta \nu}^{\prime}(z)$ at $z=0$ and $z=L$. It can be shown after tedious algebra that the transmission probabilities for $f_{\eta=+1, \nu}(z)$ and $f_{\eta=-1, \nu}(z)$ states are equal. Therefore, it is necessary to discuss only the case of $f_{\eta=+1, \nu}(z)$ explicitly.

Since the transformation (3.2) from $\phi$ to $\psi$ is through an operator $\left[\left(p_{0}-A_{0}(z)\right) \beta-\gamma\right.$. $\boldsymbol{p}+m(r)]$, which contains the operator " $\partial / \partial z$ ", the continuity of the wave function $\psi$ at $z=0$ and $z=L$ will require continuity for both $f_{\eta \nu}(z)$ and $f_{\eta \nu}^{\prime}(z)$ at these boundaries.

Making the transformation from $z$ to $\xi$ by $\xi=(\kappa z+E) \sqrt{2 / \kappa}$, the longitudinal solutions in Regions I and III are just harmonic waves. For Region II, the longitudinal equation (3.7) becomes

$$
\left\{-\frac{d^{2}}{d \xi^{2}}+\left(a+\eta \frac{i}{2}\right)-\frac{1}{4} \xi^{2}\right\} f_{\eta \nu}(\xi)=0
$$

with

$$
a=\frac{m_{\perp}{ }^{2}}{2 \kappa} .
$$


Eqs. (3.10) and (3.11) are identical to Eqs. (3.9) and (2.8) of I. Hence, the solutions in I can be used directly here by making the replacement

$$
\left.m^{2}+p_{x}^{2}+p_{y}^{2} \text { (of Ref. } 3\right) \rightarrow m_{\perp}^{2},
$$

as shown by Pavel and Brink [9].

\section{THE PAIR-PRODUCTION PROBABILITY}

A particle-antiparticle pair is spontaneously produced in the strong field when a particle in a negative-energy state $E$ with a momentum $-\left|p_{z}\right|$ tunnels from the negative energy continuum to the positive energy one [3]. By choosing the incident amplitude $I$ to be unity, the transmission amplitude $T(E)$ is the probability amplitude for the transmission of a particle from Region I to Region III with the accompanying creation of an antiparticle in Region I. For an incident particle with an energy $E$ the probability for a pair production is then $|T(E)|^{2}$. The threshold flux tube length, below which no pair production can take place, is $2 m_{\perp} / \kappa$. Because $m_{\perp}$ is greater than the rest mass $m_{0}$ due to the zero-point motion, the threshold length of the flux tube increases as a result of transverse confinement. For a given length $L$, we need to consider only a finite number of transverse excited states with $m_{\perp} \leq \frac{1}{2} k L$. The longer the flux tube, the greater will be the number of transverse states that can be produced.

In the case without a boundary, the transverse mass $m_{\perp}^{2}=m_{0}^{2}+\boldsymbol{p}_{\perp}^{2}$ is a continous variable, but in a flux tube the transverse mass assumes discrete values. Therefore, in evaluating the pair production rate the phase-space integral in the transverse direction,

$$
A \sum_{\text {spin }} \int \frac{d^{2} p_{\perp}}{(2 \pi)^{2}},
$$

with the transverse area $A$, is replaced by a summation over the finite number of transverse states, $\sum_{\eta \nu}$. For each transverse state, the integral in the longitudinal momentum is restricted by $E=-\sqrt{p_{z}^{2}+m_{\perp}^{2}} \geq m_{\perp}-k L$, or

$$
0 \leq\left|p_{z}\right| \leq \sqrt{k L\left(k L-2 m_{\perp}\right)}
$$


With the replacement of the continuous integral in $\boldsymbol{p}_{\perp}$ by the discrete summation in Eq. (4.12) in [3], the particle production rate becomes [17]

$$
\frac{\Delta N}{\Delta t}=\sum_{\eta \nu} \int r d r d \varphi \int_{0}^{\sqrt{k L\left(k L-2 m_{\perp}(\nu)\right)}} \frac{p_{z} d p_{z}}{2 \pi E} \psi_{\eta \nu}^{\dagger}(r, \varphi, z) \psi_{\eta \nu}(r, \varphi, z) \frac{v_{R}}{v_{L}}\left|T_{\nu}^{(\eta)}(E)\right|^{2}
$$

where

$$
\psi_{\eta \nu}(r, \varphi)=[E \beta-\boldsymbol{\gamma} \cdot \boldsymbol{p}+m(r)] \phi_{\eta \nu}(r, \varphi) a_{I} e^{-i k_{L} z}
$$

Since $\phi_{\eta \nu}(r, \varphi)$ is properly normalized, and $\psi_{\eta \nu}^{\dagger}(r, \varphi, z) \psi_{\eta \nu}(r, \varphi, z)$ is independent of $z$, we have

$$
\int r d r d \varphi \psi_{\eta \nu}^{\dagger}(r, \varphi, z) \psi_{\eta \nu}(r, \varphi, z)=\int r d r d \varphi \phi_{\eta \nu}^{\dagger}(r, \varphi) \phi_{\eta \nu}(r, \varphi)=1
$$

Therefore, we have

$$
\frac{\Delta N}{\Delta t}=\sum_{\eta \nu} \int_{0}^{\sqrt{k L\left(k L-2 m_{\perp}(\nu)\right)}} \frac{p_{z} d p_{z}}{2 \pi E} \frac{v_{R}}{v_{L}}\left|T_{\nu}^{(\eta)}(E)\right|^{2}
$$

These limits are important for a numerical calculation, especially for the rate near the threshold or for high transverse excitations. In the continuous limit where the transverse area $A$ is large, the formula for the rate of pair production given in Ref. [3] is recovered except for the explicit limits on the integral of $p_{z}$.

\section{CONCLUSIONS AND DISCUSSIONS}

It was recognized by Pavel and Brink for an infinitely long flux tube and fermion pairs that the pair production probability for the case with transverse confinement can be obtained by replacing the continuous mass with a discrete set of $m_{\perp}$ [9]. Generalizing this result to the case with a finite longitudinal length, for both fermion and boson pairs, the pair production probability are just given by previous results obtained in I, with the replacement of the continuous transverse mass $m_{\perp}$ by a set of discrete $m_{\perp}$ obtained in an eigenvalue equation in the transverse degrees of freedom. A different transverse excitation is represented by a different mass $m_{\perp}$ which contains contributions from the rest mass (current mass) and the 
additional zero-point energy arising from tranverse confinement. Pair production occurs only when the longitudinal separation is greater than the threshold length $2 m_{\perp} / \kappa$ which increases as a result of transverse confinement. Furthermore, the pair-production probability is much suppressed when the separation just exceeds the threshold value. The probability also shows oscillations due to the finite separation [3]. In contrast, in the case without transverse confinement, the mass which needs to tunnel through the barrier in the longitudinal direction

$m_{\perp}=\sqrt{m_{0}^{2}+p_{T}^{2}}$ is a continuous variable. The lowest transverse mass with $p_{T}=0$ is just the rest mass of the particle $m_{0}$.

The above results shed light on the values of masses one should use in the application of the Schwinger mechanism to examine $q \bar{q}$ production [2, [4]. There, it is often a question whether one should use the current quark mass (of a few $\mathrm{MeV}$ for $u$ and $d$ quarks) or the constituent quark mass (which is of the order of a few hundred MeV), for the threshold of pair production. Our analysis of the transverse boundary indicates that the proper mass one should consider for the Schwinger mechanism is neither the current mass nor the constituent mass but the transverse mass $m_{\perp}$ which depends on the current quark mass $m_{0}$, the finite transverse dimension of the flux tube, and the transverse state quantum number $\nu$ of the particle. It includes the zero-point energy of the transverse motion, which is of the order of $\hbar /$ (flux tube radius). With a flux tube radius of the about $0.5 \mathrm{fm}$, the transverse zero-point energy is of the order of a few hundred MeV. The transverse mass $m_{\perp}$ is therefore about a few hundred $\mathrm{MeV}$, for the production of particles in the lowest transverse state.

\section{ACKNOWLEDGMENTS}

The authors would like to thank Dr. G. Gatoff for helpful discussions. This research was supported in part by the Division of Nuclear Physics, U.S. Department of Energy under Contract No. DE-AC05-84OR21400 managed by Martin Marietta Energy Systems, Inc., and in part under Contract No. DE-FG05-94ER40883. 


\section{REFERENCES}

[1] J. Schwinger, Phys. Rev. 82, 664 (1951).

[2] A. Casher, H. Neuberger, and S. Nussinov, Phys. Rev. D20, 179 (1979).

[3] R. C. Wang and C. Y. Wong, Phys. Rev. D38, 348 (1988) and references cited therein.

[4] C. Y. Wong, Introduction to High-Energy Heavy-Ion Collisions, World Scientific Publishing Company, Singapore, 1994.

[5] X. Artru and G. Mennessier, Nucl. Phys. B70, 93 (1974); X. Artru, Phys. Rep. 97, 147 (1983).

[6] B. Andersson, G. Gustafson, G. Ingelman, and T. Sjötrand, Phys. Rep. 97, 31 (1983).

[7] N. K. Glendenning and T. Matsui, Phys. Rev. D28, 2890 (1983).

[8] C. Martin and D. Vautherin, Phys. Rev. D38, 3953 1988; and ibid. D40, 1667 (1989).

[9] H. P. Pavel and D. M. Brink, Z. Phys. C51, 119 (1991).

[10] K. Sailer et al., Phys. Lett. B240, 381 (1990).

[11] Th. Schönfeld et al., Phys. Lett. B247, 5 (1990)

[12] G. Gatoff and C. Y. Wong, Phys. Rev. D46, 997 (1992).

[13] C. S. Warke and R. S. Bhalerao, Pramana - J. Phys. 38, 37 (1992).

[14] C. S. Warke and R. S. Bhalerao, Phys. Rev. D46, 5168 (1992).

[15] I. D. Flintoft and M. C. Birse, J. Phys. G. 19, 389 (1993).

[16] M. Abramowitz and I. A. Stegun, Handbook of Mathematical Functions, Dover Publications, New York, 1972.

[17] In Ref. [3], Eq. (4.11) has a misprint; the expression " $\left|\mathbf{J}_{T}\right| /\left|\mathbf{J}_{L}\right|$ " on the left hand side should read " $\left|\mathbf{J}_{T}\right| /\left|\mathbf{J}_{I}\right|$ ", where the subscript $I$ stands for the incident current. 